\title{
Iso-energy-efficiency: An approach to power-constrained parallel computation
}

\author{
Shuaiwen Song ${ }^{1}$, Chun-Yi Su${ }^{1}$, Rong $\mathrm{Ge}^{2}$, Abhinav Vishnu ${ }^{3}$, and Kirk W. Cameron ${ }^{1}$ \\ ${ }^{1}$ SCAPE Laboratory, Virginia Tech ${ }^{2}$ Marquette University ${ }^{3}$ Pacific Northwest National Laboratory \\ s562673@vt.edu,sonicat@vt.edu,rong.ge@marquette.edu,Abhinav.Vishnu@pnl.gov,cameron@vt.edu
}

\begin{abstract}
Future large scale high performance supercomputer systems require high energy efficiency to achieve exaflops computational power and beyond. Despite the need to understand energy efficiency in high-performance systems, there are few techniques to evaluate energy efficiency at scale. In this paper, we propose a system-level iso-energy-efficiency model to analyze, evaluate and predict energy-performance of data intensive parallel applications with various execution patterns running on large scale poweraware clusters. Our analytical model can help users explore the effects of machine and application dependent characteristics on system energy efficiency and isolate efficient ways to scale system parameters (e.g. processor count, CPU power/frequency, workload size and network bandwidth) to balance energy use and performance. We derive our iso-energy-efficiency model and apply it to the NAS Parallel Benchmarks on two power-aware clusters. Our results indicate that the model accurately predicts total system energy consumption within 5\% error on average for parallel applications with various execution and communication patterns. We demonstrate effective use of the model for various application contexts and in scalability decision-making
\end{abstract}

Keywords: Iso-energy-efficiency, Performance Isoefficiency, Power Con sumption, Power-Aware Clusters.

\section{INTRODUCTION}

As we enter the era of exascale computing, energy consumption of large scale parallel systems and data centers has become one of the most significant hindrances for designing highly scalable data intensive applications and larger parallel systems. For instance, recommendations in a recent report from the US Department of Energy suggest the power consumption of an exaflop machine, capable of a 1000-fold performance increase over current petaflop systems, must be constrained to a 10-fold increase in power consumption [1]. This engineering challenge coupled with the high operational costs and system failure rates associated with many-megawatt computing resources has increased the need to consider power and the entangled effects of performance in emergent exascale systems and applications.

Research[2-5] in high-performance power-aware computing has focused on identifying power saving opportunities in communication phases and applying DVFS [6] (dynamic voltage and frequency scaling) strategies to these phases to reduce power consumption without sacrificing performance. Figure 1 depicts the types of controllers used in these techniques to build sophisticated power management software. The focus in previous work has been developing a controller that uses observational data and (in later techniques) predictive data to schedule power states and balance performance.

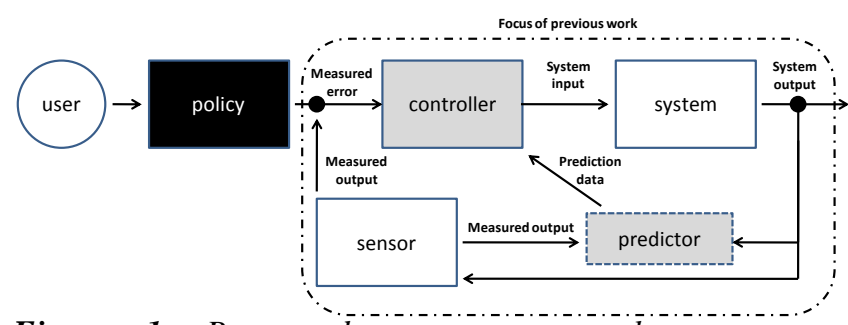

Figure 1. Past and current approaches to power management in high-performance systems.

A key limitation of past approaches is a lack of powerperformance policies allowing users to quantitatively bound the effects of power management on the performance of their applications and systems. Existing controllers and predictors use policies fixed by a knowledgeable user to opportunistically save energy and minimize performance impact. While the qualitative effects are often good and the aggressiveness of a controller can be tuned to try to save more or less energy, the quantitative effects of tuning and setting opportunistic policies on performance and power are unknown. In other words, the controller will save energy and minimize performance loss in many cases but we have little understanding of the quantitative effects of controller tuning. This makes setting power-performance policies a manual trial and error process for domain experts and a black art for practitioners. To improve upon past approaches to high-performance power management, we need to quantitatively understand the effects of power and performance at scale.

We use a modeling based approach that captures powerperformance tradeoffs system-wide and at scale. Our basic idea is to apply the concept of iso-efficiency [7] for performance, or the ability to maintain constant per-node performance as a system scales, to power-performance management. We want to create techniques that allow us to quantitatively control and maintain power-performance as systems and applications scale; we thus name our approach iso-energy-efficiency. In conducting this work, we found the first essential step toward controlling for iso-energyefficiency was to create a detailed, sophisticated, accurate model of the effects of performance and power on scaled systems and applications.

The contributions of this work include: 
- Develop ment of a fine-grained, analytical iso-energyefficiency model that incorporates parallel system components and computational overlap at scale.

- Accuracy analysis and verification of the model on two power-scalable clusters.

- Creation of a set of open source tools for deriving and measuring model input parameters.

- Results from a detailed power-performance scalability analysis of EP, FT and CG from the NAS Parallel Benchmarks [8], including use of the iso-energyefficiency model to bound and maintain systemenergy efficiency at scale.

To the best of our knowledge, this is the first systemlevel, scalable, analytical model of both power and performance on real systems and applications. We begin the succeeding discussions with some related work followed by an overview of the model. Next, we show validation and results using the model to perform scalability analysis of the NAS Parallel Bench marks. Lastly, we show full derivation of the model and its parameters and conclusions.

\section{RELATED WORK}

\section{A. Isoefficiency}

According to Amdahl's law [9], speedup for parallel systems is limited by the amount of paralle lis $m$ inherent in the application. This law characterizes the performance impact of parallelism. Though there are several other alternative vie wpoints on speedup, the most relevant to our work is that of Grama et al [7] who proposed a formal performance isoefficiency function describing how ideally performance efficiency will remain constant relative to the smallest node configuration.

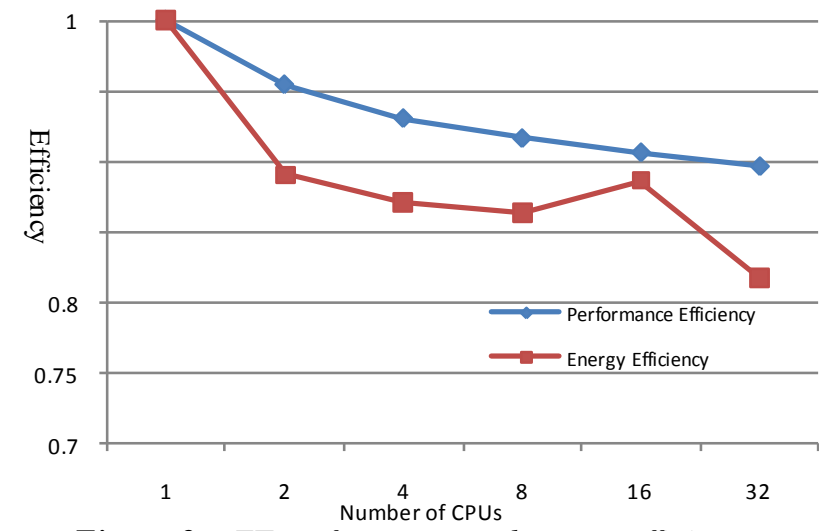

Figure 2a. FT performance and energy efficiency.

For a fixed problem size, Figures $2 \mathrm{a}$ and $2 \mathrm{~b}$ show the performance efficiency curves for FT and CG. FT scales reasonably well while CG drops off at 16 CPUs then recovers relative to the ideal case. There are a plethora of performance analysis tools and techniques available to help us interpret and understand an application's scalability. These analyses may suggest any number of root causes that can be addressed to improve isoefficiency.

In contrast, just measuring energy use is challenging for non experts. Figures $2 \mathrm{a}$ and $2 \mathrm{~b}$ show the energy efficiency for FT and CG. Moreover, even though the energy efficiency (or lack thereof) in these applications is obvious as they scale, there are few tools currently available to explain the observed energy efficiency.

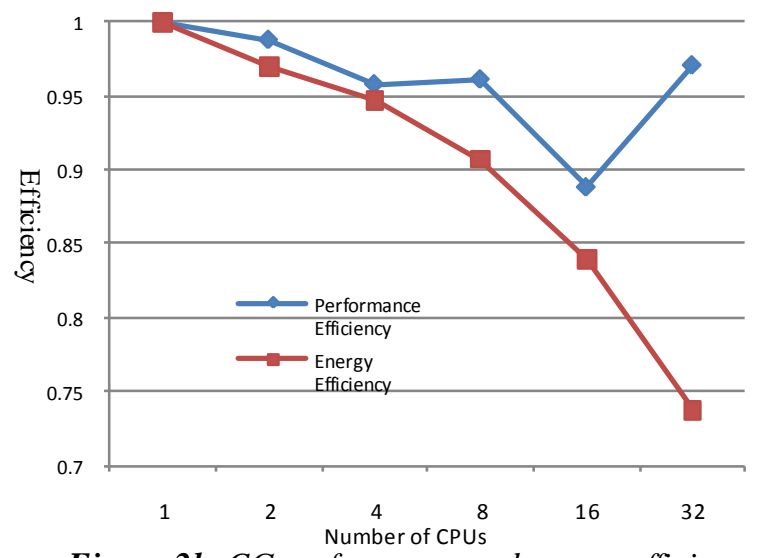

Figure $2 b$. CG performance and energy efficiency.

Being able to identify the root cause of energy inefficiency would allow us to improve system and application efficiency more in line with the ideal isoefficient case. However, analyzing and potentially predicting energy efficiency is exceedingly difficult since we must identify and isolate the interacting effects of power and performance. For example, changing the power settings on a processor using DVFS affects performance which in turn potentially affects the length of time an application takes to complete which is key to its overall energy usage.

\section{B. Parallel performance models}

There has been extensive research conducted on performance speedup and scalability of parallel applications in high performance computing. As mentioned, Amdahl's law [9] introduced the concept that the speedup is limited by the fraction of the workload that can be computed in parallel. Grama et al [7, 10] formally defined isoefficiency as discussed. The fixed-time speedup model [11], memorybounded speedup model [12], and other related studies[13, 14] all extend A mdahl's law in unique ways. However, all of these approaches focus on performance and ignore both energy consumption and the performance effects of power management.

\section{Energy efficiency in HPC}

Several high-profile efforts such as the Top500 List [15], the Green 500 List [16], the SPECPower benchmark [17], and power-performance evaluation of the HPCC benchmarks $[18,19]$ have elevated the interest in energy efficiency for high-end systems and servers. 
Ge et al proposed the PowerPack [20] framework for measuring correlated power and performance data on large scale systems and we use this framework to collect the results presented. Early work to improve the efficiency of high-end systems [3, 4, 21, 22] used various DVFS scheduling strategies to gain significant energy savings under performance constraints. Freeh et al [2, 23, 24] similarly studied energy-performance tradeoffs for MPI applications.

Our proposed iso-energy-efficiency model analyzes and predicts the combined effects of performance and power on scalable systems. The policy module highlighted in Figure 1 is a practical application of improved understanding of the power-performance tradeoffs and contrasts our work with approaches to energy efficiency in HPC which have historically focused on improving controllers and predictors. The iso-energy-efficiency approach will improve our understanding of power-performance to quantitatively bound the impact of power manage ment on performance.

\section{Energy modelling}

The power-aware speedup model proposed by Ge and Cameron [25] is a generalization of Amdahl's Law for energy. While this model accurately captures some of the effects of energy management on speedup, it provides little insight to the root cause of poor power-performance scalability.

In contrast, the iso-energy-efficiency model generally predicts energy consumption as the system scales up allowing direct analysis and comparison of the tradeoffs between various model parameters.

The Energy Resource Efficiency (ERE) metric proposed by Jiang et al [26] defines a link between performance and energy variations in a system to clearly highlight the various performance-energy tradeoffs. As with other models that identify energy efficiency, this model analyzes at a very high-level and does not identify causal relationships with poor metric results.

The energy model proposed by Ding et al [27] uses circuit-level simulation to analyze power-performance tradeoffs. While this model shows promise for circuit-level design, it is too unwieldy for use in analy zing existing largescale power-scalable clusters. The model also makes a number of simplify ing assumptions such as homogenous workloads and no computational overlap making it less practical for modeling real systems.

\section{ISO-ENERGY-EFFICIENCY MODEL}

Here we briefly describe the iso-energy-efficiency model for evaluating the power-performance tradeoffs of parallel applications and systems. The derivation of the model is described in detail in Section 6. Tables 1 and 2 provide a summary of all model para meters.

Let $E_{1}$ be the total energy consumption of sequential execution and $E_{p}$ be the total energy consumption of parallel execution for a $\mathrm{g}$ iven application on $p$ parallel processors.
Table 1 Machine-depended parameters

\begin{tabular}{|c|l|}
\hline Parameters & Definition \\
\hline $\mathrm{W}_{\mathrm{c}}$ & Total on-chip computation workl oad \\
\hline $\mathrm{W}_{\mathrm{m}}$ & Total off-chip memory access workload. \\
\hline $\mathrm{W}_{\mathrm{co}}$ & $\begin{array}{l}\text { Total parallel computation overhead } \\
\text { Total number of memory access overhead } \\
\text { in parallelization }\end{array}$ \\
\hline $\mathrm{W}_{\mathrm{mo}}$ & $\begin{array}{l}\text { Total number of messages packaged in } \\
\text { parallelization }\end{array}$ \\
\hline $\mathrm{M}$ & $\begin{array}{l}\text { Total number of bytes transmitted } \\
\text { fumber of homogeneous processors available } \\
\text { for computing the workloads }\end{array}$ \\
\hline $\mathrm{P}$ & $\begin{array}{l}\text { Workload or total amount of work(in } \\
\text { instructions or computations) }\end{array}$ \\
\hline$\alpha$ & $\begin{array}{l}\text { the extent of overlap among computation, } \\
\text { memory access and network transmission }\end{array}$ \\
\hline$T_{o}$ & $\begin{array}{l}\text { Total overhead time due to parallelism } \\
\text { running on a single processor }\end{array}$ \\
\hline$T_{1}$ &
\end{tabular}

Table 2 Application -depen ded parameters

\begin{tabular}{|c|c|}
\hline parameters & Definition \\
\hline \multicolumn{2}{|c|}{ Time related } \\
\hline $\mathrm{t}_{\mathrm{c}}$ & $\begin{array}{l}\frac{C P I_{\text {on }}}{f}[28], \text { Average time per on-chip } \\
\begin{array}{l}\text { computation instruction } \\
\text { caches and registers) }\end{array}\end{array}$ \\
\hline$t_{m}$ & Average memory access latency \\
\hline$t_{\mathrm{msg}}$ & Average start up time to send a message \\
\hline $\mathrm{t}_{\text {Byte }}$ & Average time of transmitting a 8-bits word \\
\hline $\mathrm{T}_{\mathrm{IO}}$ & Total I/O access time \\
\hline \multicolumn{2}{|c|}{ Power-related } \\
\hline$P_{c-o n}$ & Average CPU power in running state \\
\hline $\mathrm{P}_{\mathrm{c}-\mathrm{idle}}$ & Average CPU power in idle state \\
\hline$\Delta \mathrm{P}_{\mathrm{c}}$ & $\mathrm{P}_{\mathrm{c}-\text { on }}-\mathrm{P}_{\mathrm{c}-\text { idle }}$ \\
\hline $\mathrm{P}_{\mathrm{m}-\mathrm{on}}$ & Average memory power in running state \\
\hline $\mathrm{P}_{\mathrm{m}-\mathrm{idle}}$ & Average memory power in idle state \\
\hline$\Delta \mathrm{P}_{\mathrm{m}}$ & $\mathrm{P}_{\mathrm{m}-\text { on }}-\mathrm{P}_{\mathrm{m} \text {-idle }}$ \\
\hline $\mathrm{P}_{\mathrm{IO}-\mathrm{on}}$ & Average IO device power in running state \\
\hline $\mathrm{P}_{\mathrm{IO}-\text { idle }}$ & Average IO device power in idle state \\
\hline$\Delta \mathrm{P}_{\mathrm{IO}}$ & $\mathrm{P}_{\mathrm{IO}-\text { on }}-\mathrm{P}_{\mathrm{IO}-\text { idle }}$ \\
\hline $\mathrm{P}_{\text {other }}$ & $\begin{array}{l}\text { Average sum of other devices' power such } \\
\text { as motherboard, System/CPU fans, NIC, etc. }\end{array}$ \\
\hline $\mathrm{P}_{\text {total-idle }}$ & Average system power on idle state \\
\hline$f$ & $\begin{array}{l}\text { The clock frequency in clock cycles per } \\
\text { second }\end{array}$ \\
\hline
\end{tabular}

Let $E_{o}$ represent the additional energy overhead required for parallel execution:is the energy overhead for parallel execution.

$$
E_{o}=E_{p}-E_{1}
$$

We now define iso-energy-efficiency (EE) as:

$$
E E=\frac{E_{1}}{E_{p}}=\frac{E_{1}}{E_{1}+E_{o}}=\frac{1}{1+E_{o} / E_{1}}
$$


Let $\mathrm{EEF}=\frac{E_{o}}{E_{1}}$ be the energy efficiency factor $(E E F) . E E F$ is the ratio of parallel energy overhead to the energy of an application running sequentially. An application with a large $E E F$ has low energy efficiency, and vice versa. Effective use of the iso-energy-efficiency model (EE) requires accurate estimation of the EEF. We can more accurately estimate EEF using the following equation:

$E E F=\frac{E_{O}}{E_{1}}=\frac{\alpha T_{o} P_{\text {total-idle }}+W_{c o} t_{c} \Delta P_{c}+W_{m o} t_{m} \Delta P_{m}}{\alpha T_{1} P_{\text {total-idle }}+W_{c} t_{c} \Delta P_{c}+W_{m} t_{m} \Delta P_{m}}$

$E E$ then becomes:

$E E=\frac{1}{1+E_{o} / E_{1}}=\frac{1}{1+E E F}$
$=1 /\left(1+\frac{\alpha T_{o} P_{\text {total-idle }}+W_{c o} t_{c} \Delta P_{c}+W_{m o} t_{m} \Delta P_{m}}{\alpha T_{1} P_{\text {total-idle }}+W_{c} t_{c} \Delta P_{c}+W_{m} t_{m} \Delta P_{m}}\right)$

Equations (3) and (4) form the basis for computing isoenergy-efficiency. The challenge is to capture each of the parameters used in these equations for a given application and system co mbination.

Tables 1 and 2 show the model parameters used to calculate EEF and EE can be classified as either machinedependent or application-dependent. The machinedependent variable vector can be described as a function of frequency (i.e. computational speed) and workload bandwidth (i.e. co mputational throughput) of the hardware:

$$
\begin{aligned}
& V_{\text {machine }}\left(f, N_{\text {bandwidth }}\right) \\
& =\left(t_{c}, t_{m}, t_{\text {ms } g}, t_{\text {Byte }}, P_{\text {total-idle }}, \Delta P_{c}, \Delta P_{m}\right)
\end{aligned}
$$

The application-dependent variable vector can be described as a function of the amount of parallelism available and the workload for the application:

$$
V_{a p p}(p, n)=\left(\alpha, W_{c}, W_{m}, W_{c o}, W_{m o}, M, B\right)
$$

Section 6 provides details describing and motivating the use of these parameters. The reader may skip to this section to learn more about the iso-energy-efficiency model derivation or continue to the next two sections where we validate the iso-energy-efficiency model and demonstrate its usefulness for evaluating parallel power-performance efficiency.

\section{TEST ENVIRONMENT AND MODEL VALIDATION}

\section{A. Test Environment}

We use two different power-aware clusters to conduct our experiments: SystemG and Dori. The SystemG 22.8 TFlop supercomputer provides a research platform for development of high-performance software tools and applications at scale. It utilizes $325 \mathrm{Mac}$ Pro computer nodes and each node has two 4-core $2.8 \mathrm{Gh}$ z Intel Xeon Processors.
Each node has an 8 GB RAM and each core has a 6 MB cache. SystemG is equipped with Mellanox 40Gbytes/sec end to end InfiniBand adapters and switches which dramatically increases the transmission bandwidth and reduce the latency. Since G stands for 'green", SystemG is a power-scalable system and has over 10,000 power and thermal sensors. DVFS, concurrency throttling and dynamic thermal monitoring enabled. Intelligent Power Distribution Units (Dominion PX) are attached to adjacent machines so users can dynamically profile power consumption of controlled machines or re motely turn on/off nodes, etc.

The Dori system is composed of 8 nodes and each node contains dual core AMD Opteron Process or dusl processors. Each node has 6 GB RAM and each core has 1 MB cache. Dori is equipped with 1 Gbytes/sec Ethernet and switches.

PowerPack 2.0 [18, 20], designed and implemented by the SCAPE Laboratory at Virginia Tech, is a framework for power/energy profiling, analysis and prediction of parallel applications and systems. The PowerPack infrastructure is composed of both hardware and software components: the hardware is responsible for accurate and reliable direct measurement of both system-wide and component level power consumption and the software automatically collects, processes and synchronizes power data with system load. We used the PowerPack toolkit for all of the power and performance measurements obtained herein on both clusters.

The NAS Parallel Bench marks consist of 5 kernels and 3 pseudo-applications that mimic the computation and data movement characteristics of large scale CFD applications which are widely used in HPC community. We validate the proposed model on both systems for the NAS Parallel Benchmarks. We conducted scalability studies for 3 benchmarks (FT, CG, EP) on SystemG.

\section{B. Model Validation}

To validate the iso-energy-efficiency model, we need to verify the correctness of the model single and parallel processor configurations. We vigorously measure and derive the parameters from Tables 1 and 2; namely the machine and application dependent parameters.

For the machine-dependent parameters, we built a tool using the Perfmon API from UT-Knoxville to automatically measure the average $t_{c}$ (time per on-chip computation instruction) derived as $\frac{C P I_{o n}}{f}$. We use the lat_mem_rd function from the LMbench microbenchmark [29] to estimate memory costs $t_{m}$. $t_{m s g}$ and $t_{B y t e}$ is obtained by using the MPPTest tool [30] for both the InfiniBand [31] and Ethernet interconnects in the two clusters. In addition, $P_{\text {total-idle }}, \Delta P_{c}$ and $\Delta P_{m}$ can be obtained by using PowerPack [20]. We did not include disk $\mathrm{I} / \mathrm{O}$ in our estimations for our energy efficiency model because the applications we tested are not disk intensive. We leave this to future work. For completeness, though it is not used in the current study, we were able to estimate $T_{I O}$ can be estimated by using the Linu x pseudo file /proc/stat.

For the application-dependent parameters, we build a workload and overhead model for each parameter by 
analyzing the algorith $m$ and measuring the actual workload for each application. We use Perfmon to measure each workload parameter, $W_{c}, W_{m}, W_{c o}, W_{m o}$ and we use the TAU performance tool from the University of Oregon to measure $M$ and $B$. Figure 3 illustrates the accuracy of the energy model for P processors. (Note: Specifically, these results are for Equation (15) in the derivation Section 6).

Figure 3 compares the energy consumption predicted by the iso-energy-efficiency model with the actual energy consumption obtained using the PowerPack framework on Dori for $\mathrm{p}=4$. We repeated all experiments five times to reduce measuring errors. The results indicate that the proposed energy model can accurately predict the actual energy consumption within $5 \%$ prediction error. We conducted similar experiments on SystemG. for $p=1,2,8$, 16, 32, 64, 128. Figure 4 shows the average error rate of EP, FP, and CG applications on SystemG under different levels of parallelism using the InfiniBand interconnect. The results

\section{Energy Model Validation on Dori System}

Actual Measurement (Joule) Estimation By Energy Model(Joule)

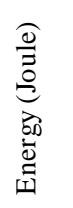

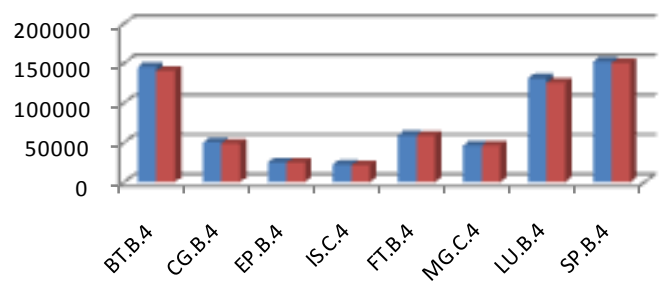

\section{NAS benchmark suites}

Figure 3. Model validation on Dori system. All the applications run on 4 nodes under same CPU clock frequency. Model accuracy for all the benchmarks are over $95 \%$.

show good accuracy. Upon detailed analysis, the relatively higher errors $(8.31 \%)$ found with $\mathrm{CG}$ were due to inaccuracies in our memory model for this application. Improving the accuracy for $\mathrm{CG}$ is the subject of future work.

Based on the accuracy results for both System $G$ and Dori clusters, we conclude that our iso-energy-efficiency model performs well on different network interconnection infrastructures and can predict total system energy consumption with an average of $5 \%$ prediction error rate for parallel applications with various execution and communication patterns.

\section{EXPERIMENTAL RESULTS}

\section{A. Energy consumption and efficiency prediction for large scale systems}

Given the accuracy of our modeling techniques as described in the previous section, we use measurements from smaller configurations to predict and analyze power- performance tradeoffs on larger systems. (Note: we build our energy consumption and efficiency models using Equations (13), (15), (18), (21) from Section 6 applied a smaller representative portion of a large scale system.

Initially, we obtain machine-dependent variables from the smaller system and use these values and our models to predict values for increasing number of nodes:

$$
\begin{aligned}
& V_{\text {machine }}\left(f, N_{\text {bandwidt }}\right) \\
& =\left(t_{c}, t_{m}, t_{\text {msg }}, t_{\text {Byte }}, P_{\text {total-idle }}, \Delta P_{c}, \Delta P_{m}\right)
\end{aligned}
$$

\section{Average error rate on SystemG}

$(\mathrm{P}=1,2,4,8,16,32,64,128)$

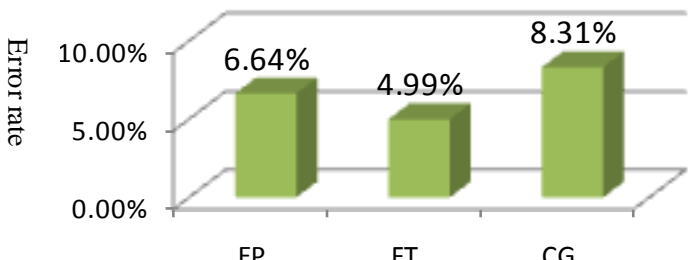

Figure 4. The average error rate of EP, FT and $C G$ program. class $=B$ in node number $p=1,2,4,8,16$, $32,64,128$.

All variables can be measured as described in the previous section. Frequency-dependent variables can be combined by normalizing measurements obtained through the use of hardware counters, LMbench, MPPTest and Powerpack. For example, $t_{c}$ can be described as $\frac{11.9}{f} \times 10^{-10} \mathrm{sec}$ on SystemG. We assume power is proportional to $f^{\gamma}(\gamma \geqslant 1)$.

Next, we model application-dependent variables from the smaller system:

$$
V_{a p p}(p, n)=\left(\alpha, W_{c}, W_{m}, W_{c o}, W_{m o}, M, B\right)
$$

Except for $\alpha$, all of these variables in $V_{a p p}(p, n)$ depend on a performance model and can be described as a function of problem size, $n$, and the level of paralle lis $\mathrm{m}, p$. For example, $W_{\text {co }}$ could be described as $n \log _{2} p$ in one-dimensional, unordered and radix-2 binary exchange Fast Fourier Transform. With all parameters accounted for, we can solve for Equations (3) and (4). (Note: Specifically, we first solve Equations (13), (15), (18), and (21) described in the next section.) We can then project values for larger values of $p$ to predict the power-performance behavior and tradeoffs of large scale systems.

\section{B. Scalability studies for NAS PB}

In this section, we analyze the power-performance characteristics of FT, EP and CG using the iso-energyefficiency approach. We isolate power-performance efficiency problems and use the model findings to tune parameters such as problem size, $n$, CPU clock frequency, $f$, and level of parallelis $\mathrm{m}, p$ to improve efficiency. 
In each case, we use the methods described in the previous sections to obtain model para meters and build our model from measurements on a smaller system. Once we've identified estimates for $V_{\text {machine }}\left(f, N_{\text {bandwidth }}\right)$ and $V_{a p p}(p, n)$ vectors, we build $E E$ and EEF as described in Equations (3) and (4). In the rest of this section, all the parameterizations are obtained for the SystemG cluster though the same methodology can be applied other platforms.

\section{1) $F T$}

FT computes a 3-D partial differential equation solution using Fast Fourier Transforms. The application stresses the CPU, memory and the communication network during various phases. Parallel FT iterates through approximately four phases during the execution: computation phase 1, reduction phase, computation phase 2 and all-to-all communication. The FT benchmark is communication intensive with dominating parallel communication overhead for the all-to-all phase. FT has a large memory footprint compared to the EP (Embarrassingly Parallel) application in the NAS suite.

We use the Pairwise exchange/Hockney model [32, 33] to estimate the MPI_Alltoall operations required to solve for EE and EEF. (Note: This replaces the general approach to communication estimation described by Equation (17) in the next section.) By analyzing the FT's Alltoall communication algorithm on the architecture of the SystemG cluster, we found the Pairwise exchange/Hockney model appropriate and accurate in our validation testing. The time duration for this implementation is described as follows:

$$
T_{\text {net }}=(p-1) *(\alpha(H)+\beta(H) * H) .
$$

In the equation above, $H$ is the message size, $\alpha(H)$ is message start up time, and $\beta(H) * H$ is the transmission time. For details, please refer to the original paper [32]. We use our own measurements, MPPTest and the PowerPack frame work to obtain the machine dependent parameters:

$$
\begin{aligned}
& V_{\text {machine-FFT }}\left(f, N_{\text {bandwidth }}\right) \\
& =\left(t_{c}, t_{m}, t_{m s g}, t_{\text {Byte }}, P_{\text {total-idle }}, \Delta P_{c}, \Delta P_{m}\right) \\
& =\left(\frac{6.41}{f} * 10^{-10}, 1.12 * 10^{-7}, 2.53 * 10^{-5}, 1.82 *\right. \\
& \left.10^{-8}, 24 f^{\gamma}, 3.4 f^{\gamma}, 0.76 f^{\gamma}\right)
\end{aligned}
$$

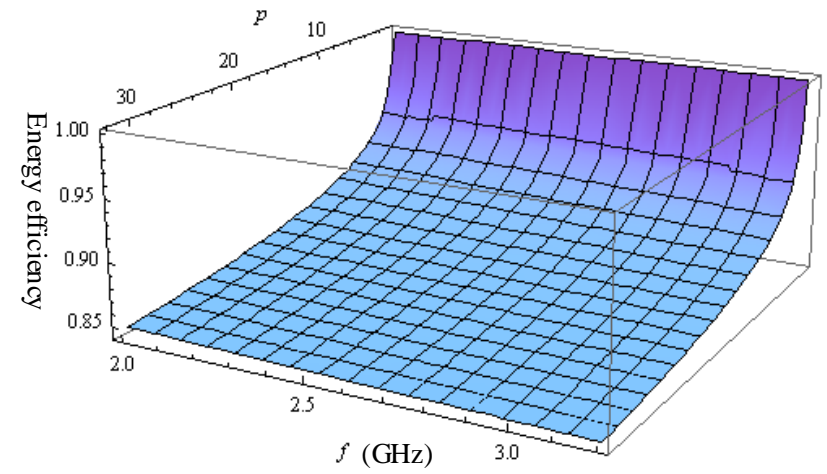

Figure 5: $3 D$ plot of $E E_{F F T}$ with $p$ and $f$ as variables.

In the equation above, for simplicity, we set $\gamma=2$ based on our test bed System G. We analyze FT and measure the actual workload by observing on-chip executing instructions, L1, L2 cache misses, main memory accesses and total instructions using Perfmon to obtain:

$V_{a p p-F F T}(p, n)=\left(\alpha, W_{c}, W_{m}, W_{c o}, W_{m o}, M, B\right.$

$=\left(0.86,1.06 * 10^{4} n, 9.49 n, 4.46 * 10^{2} n \log _{2} p,-0.73 n \log _{2} p\right.$,

$\left.22, \frac{4 n}{4^{\log p-1}}\right)$

We then solve for $E E F_{F F T}$ :

$$
\begin{aligned}
& E E F_{F F T}=\frac{E_{O}}{E_{1}}=\frac{\alpha T_{o}\left(P_{\text {total-idle }}\right)+W_{c o} t_{c} \Delta P_{c}+W_{m o} t_{m} \Delta P_{m}}{\alpha T_{1}\left(P_{\text {total }} \text { idle }\right)+W_{c} t_{c} \Delta P_{c}+W_{m} t_{m} \Delta P_{m}} \\
& =\frac{6.87 \log _{2} p-1.75 f \log _{2} p+p(p-1) f\left(\frac{11500}{n}+\frac{0.376}{4^{\log _{2} p-2}}\right)}{163+22.7 f},
\end{aligned}
$$

and thus for $E E_{F F T}$ we obtain:

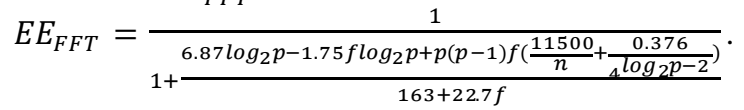

Figure 5 plots EE with a fixed workload size $n$. We can see the level of parallelism, $p$, most affects changes in energy efficiency versus frequency (or DVFS power states). In fact, for this code, frequency $f$ has little impact on energy efficiency. FT is dominated by all-to-all communications and synchronizations which makes it less likely to be influenced by changes in CPU frequency. As the number of processors scales, the effects of CPU clock frequency on onchip workload diminishes eventually while the increasing effects of parallel overhead and memory dominate. Thus, for fixed workloads on FT, increasing $p$ will dramatically decrease the energy efficiency. 


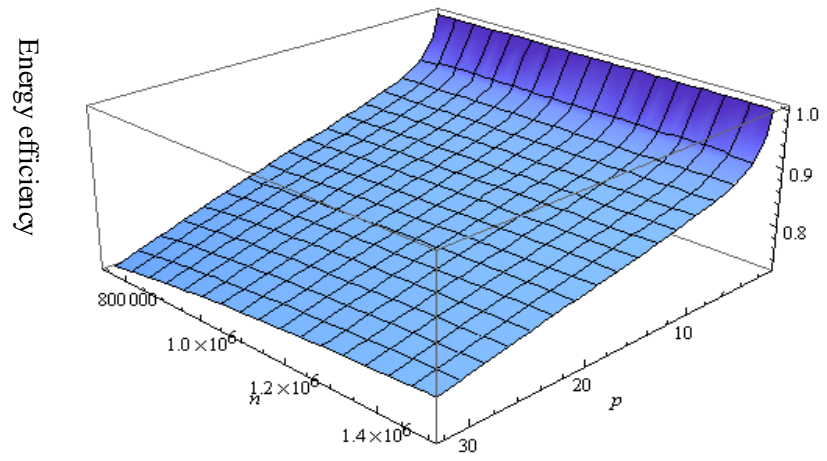

Figure 6: $3 D$ plot of $E E_{F F T}$, Assume constant frequency $f=2.8 \mathrm{GHz}$ with $p$ and $n$ as variables.

Figure 6 illustrates $E E_{F F T}$ when frequency fixes to $2.8 \mathrm{GHz}$ since frequency does not affect energy efficiency. We can see $p$ still dominates the variance of energy efficiency. It is also obvious that increasing the problem size, $n$, does enhance the energy efficiency.

\section{2) $E P$}

In parallel computing, an embarrassingly parallel $(E P)$ workload has little inter-processor communication between parallel processes. EP in the NPB benchmarks generates pairs of Gaussian random deviates using Marsaglia polar method. It separates tasks with little or no overhead. Results of EP can also be considered as a reference of peak performance of a given machine. We use our measurements, MPPTest and the PowerPack framework to obtain the mach ine dependent parameters:

$$
\begin{aligned}
& V_{\text {machine }-E P}\left(f, N_{\text {bandwidth }}\right) \\
& \quad=\left(t_{c}, t_{m}, t_{\text {ms } g}, t_{\text {Byte }}, P_{\text {total-idle }}, \Delta P_{c}, \Delta P_{m}\right) \\
& \quad=\left(\frac{11.9}{f} * 10^{-10}, 1.12 * 10^{-7}, 2.53 * 10^{-5}, 1.82 *\right. \\
& \left.10^{-8}, 18.9 f^{2}, 2.67 f^{2}, 1.52 f^{2}\right)
\end{aligned}
$$

After analyzing the parallel $E P$ codes, we have:

$$
\begin{gathered}
V_{a p p-E P}(p, n)=\left(\alpha, W_{c}, W_{m}, W_{c o}, W_{m o}, M, B\right) \\
=\left(0.93,109.4^{*} n, 1.03 * 10^{-6 * n}, 0,6.7 * 10^{-7} *^{*}(p-1), 0,0\right)
\end{gathered}
$$

Since communication in embarrassingly parallel is trivial, we simply set $M$ and $B$ to zero in $\mathrm{V}_{\text {app-EP }}(p, n)$.

Thus, from Equation (19), we have $E E F_{E P}$ :

$$
\begin{aligned}
& E E F_{E P}=\frac{E_{O}}{E_{1}}=\frac{\alpha T_{o}\left(P_{\text {total-idle }}\right)+W_{c o} t_{c} \Delta P_{c}+W_{m o} t_{m} \Delta P_{m}}{\alpha T_{1}\left(P_{\text {total-idle }}\right)+W_{c} t_{c} \Delta P_{c}+W_{m} t_{m} \Delta P_{m}} \\
& =\frac{1.43(p-1) f^{2}}{2.63 * 10^{6} f+2.2 f^{2}}
\end{aligned}
$$

So $E E_{E P}$ becomes:

$$
E E_{E P}=\frac{1}{1+\frac{1.43(p-1) f^{2}}{2.63 * 10^{6} \mathrm{f}+2.2 f^{2}}}
$$

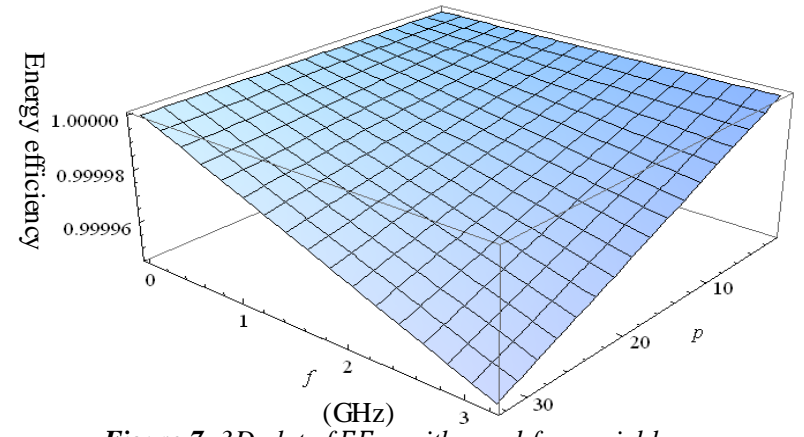

Figure $73 D$ plot of $E E_{E P}$ with $p$ and fas variables

Figure 7 illustrates the variation of $E E_{E P}$. This figure indicates that energy efficiency hardly changes with $\mathrm{p}$ and $\mathrm{f}$. Energy efficiency is close to 1 for different combinations of $\mathrm{p}$ and $f$ because only minimu m communication overhead is imposed. Since this is nearly ideal iso-energy-efficiency, we cannot improve the energy efficiency by scaling problem size $n$ at all because $E_{o}$ increases as fast as $E_{1}$.

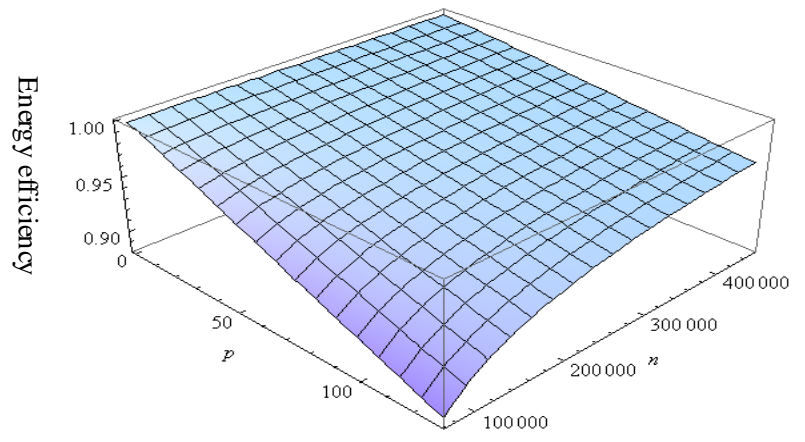

Figure $83 D$ plot of $E E_{C G}$, Assume frequency $f=2.8 \mathrm{GHz}$, with $p$ and $n$ as

\section{3) $C G$} variables.

The NAS $C G$ benchmark evaluates a parallel system's computation and communication performance. It uses the conjugate gradient method to find out the smallest eigenvalue of a large, sparse matrix. It solves a sparse linear algebra problem which is common to scientific applications on large-scale systems. We first obtain the machinedepended parameters using the previous methods:

$$
\begin{aligned}
& V_{\text {machine }-C G}\left(f, N_{\text {bandwidth }}\right) \\
& \quad=\left(t_{c}, t_{m}, t_{m s g}, t_{\text {Byte }}, P_{\text {total-idle }}, \Delta P_{c}, \Delta P_{m}\right) \\
& \quad=\left(\frac{1.14}{f} * 10^{-9}, 1.12 * 10^{-7}, 2.53 * 10^{-5}, 1.82 *\right. \\
& \left.10^{-8}, 24 f^{2}, 4.57 f^{2}, 1.25 f^{2}\right)
\end{aligned}
$$

For the application-dependent parameters we obtain:

$$
\begin{aligned}
& \quad V_{a p p-c G}(p, n)=\left(\alpha, W_{c}, W_{m}, W_{c o}, W_{m o}, M, B\right) \\
& =\left(0.85,2.13 * 10^{5} n^{1.25}, \quad 0.96 n^{1.75}, 1.86 * 10^{6} n^{0.7} *\right. \\
& 2^{\left.1.1\left(\log _{2} p-1\right),-4.75 n^{0.5} * 2^{0.14 *\left(\log _{2} P-1\right)^{2}}, 0,0\right) .}
\end{aligned}
$$

Thus, we solve for $E E F_{C G}$ : 


$$
\begin{gathered}
E E F_{C G}=\frac{E_{O}}{E_{1}}=\frac{\alpha T_{o}\left(P_{\text {total-idle }}\right)+W_{c o} t_{c} \Delta P_{C}+W_{m o} t_{m} \Delta P_{m}}{\alpha T_{1}\left(P_{\text {total-idle }}\right)+W_{c} t_{c} \Delta P_{c}+W_{m} t_{m} \Delta P_{m}} \\
= \\
\frac{5.3 * 10^{-2} f n^{0.7} * 2^{1.1}\left(\log _{2} p-1\right)-1.15 * 10^{-3} f^{2} n^{0.5} * 2^{0.14 *\left(\log _{2} P-1\right)^{2}}}{6.07 * 10^{-3} f n^{1.25}+2.33 * 10^{-6} f^{2} n^{1.75}},
\end{gathered}
$$$$
\text { and then for } E E_{C G} \text { : }
$$$$
E E_{C G}=
$$

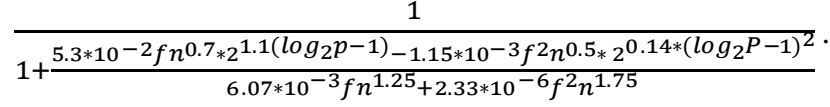

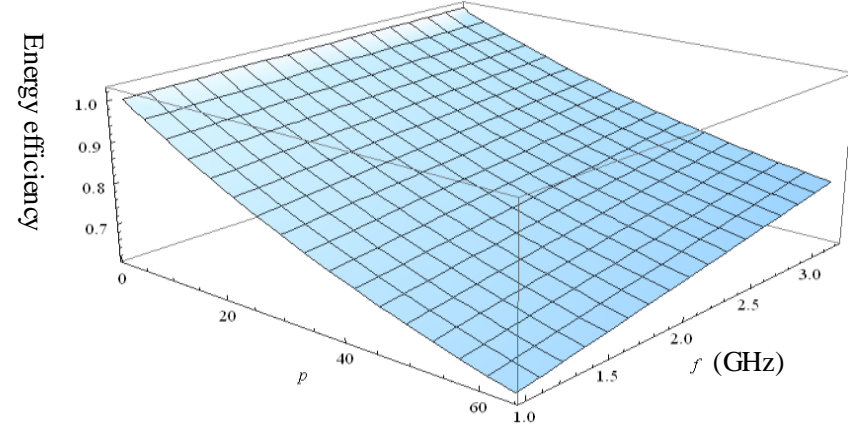

Figure 93 plot ofE $E_{C G}$, Assume problem size $n=75000$, with $p$ and $f$ as variables.

From $E E F_{C G}$ and $E E_{C G}$, we plot the relationships between level of parallelism, $p$, problem size, $n$ and frequency, $f$. In Figure 8, we first fix the frequency $f$ at 2.8 $\mathrm{GHz}$ to examine the relation between $p$ and $n$. We notice that the energy efficiency decreases as $p$ increases. However, increasing the workload size, $n$, will improve the energy efficiency.

Fixing the workload size n, we next observe the relationship between $p$ and $f$. Figure 9 shows energy efficiency declines with increase in the level of paralle lis $\mathrm{m}$. In contract to $E P$, the energy efficiency increases with CPU frequency. Digging further to examine the energy overhead $E_{o}$ and energy consumption of $E_{1}$, we observe both increase when frequency increases. However, the $E E F_{C G}$ decreases while frequency increases because $E_{1}$ increases faster than $E_{0}$. In this strong scaling case, users can scale the frequency up using DVFS to achieve better energy efficiency. Also, compared to FT (see Figure 6), the effects of frequency have more impact on the on-chip workload of CG than FT as $p$ scales due to a lower communication to computation ratio.

\section{4) Discussion of $P_{\text {total-idle }}, \Delta P_{c}, \Delta P_{m}$}

We classify $P_{\text {total-idle }}, \Delta P_{c}$ and $\Delta P_{m}$ into machinedependent variables because their behaviors are highly related to Chip's $V_{a d d}$ and frequency, $f$. However, they are not only affected by machine architecture but also affected by traits of application. The execution pattern of an application could also affect the power consumption during execution. For simplicity, we assume they are only affected by hardware. From Kim, et al [6, 34], we assume power is proportional to $f^{\gamma}(\gamma \geqslant 1)$. Different hardware architecture could result in different $\gamma$ value.
5) Discussion of the effect of the level of parallelism, $p$

We can rewrite Equation (16) as follows to see the relation between $E_{0}$ and $p$ when the workload is evenly divided among processers (homogeneous workload):

$$
\begin{aligned}
& \quad E_{O}=E_{p}-E_{1} \\
& =\alpha T_{o}\left(P_{\text {total-idle }}\right)+p w_{c o} t_{c} \Delta P_{c}+p w_{m o} t_{m} \Delta P_{m} \\
& \quad=p\left[\alpha\left(w_{c o} t_{c}+w_{m o} t_{m}+T_{n e t}\right)+w_{c o} t_{c} \Delta P_{c}+\right. \\
& \left.\quad w_{m o} t_{m} \Delta P_{m}\right] \\
& \text { where } T_{o}=\left(p w_{c o} t_{c}+p w_{m o} t_{m}+p T_{n e t}\right)
\end{aligned}
$$

Thus, $E_{0}$ is $O\left(p^{k}\right)(\mathrm{k} \geq 1)$. Generally speaking, more parallelization will incur lower energy efficiency. In this case, the application's tasks among all nodes require extra computation, memory accesses and communication efforts to coordinate with each other to complete the job. We observe this phenomenon in $F T$ and $C G$. In contrast, $E P$ incurs almost no overhead and energy efficiency doesn't decrease significantly with the increase of the levels of parallelization.

6) Discussion of problem size $n$.

Problem size is a dominant factor affecting energy efficiency. The $E E$ for applications FT and CG improve if the problem size scales. However, increasing problem size does not necessarily improve energy efficiency as in the case of EE for EP.

7) Discussion of frequency, $f$

Decreasing frequency can either increase or decrease energy efficiency. For $E P$ and $F T$, we observed no energy efficiency improvements for parallel execution when we adjust to low frequency. However, in the case of $C G$, we found that higher frequencies can improve energy efficiency because the me mory overhead $W_{\text {mo }}$ value decreases.

\section{MODEL DETAILED DERIVAT ION}

In this section we describe the details for deriving the isoenergy-efficiency model first presented in Section 3.

\section{A. Performance Model}

At the system level, the theoretical sequential execution time for an on-chip/off-chip workload comprises three components $[35,36]$ : computation time $W_{c} t_{c}$ (with on-chip instruction execution frequency), main memory access latency $W_{m} t_{m}$, and $\mathrm{I} / \mathrm{O}$ access time $T_{I O}$ (with off-chip instruction execution frequency). Thus the theoretical execution time can be expressed as:

$$
T=W_{c} t_{c}+W_{m} t_{m}+T_{I O}
$$

Since optimization techniques could raise various levels of overlap between components [37], we multiply $T$ by an overlap factor $\alpha(0 \leq \alpha \leq 1)$ such that:

$$
T^{\prime}=\alpha T=\alpha\left(W_{c} t_{c}+W_{m} t_{m}+T_{I O}\right)
$$$$
\text { where } T^{\prime} \text { is the actual execution time. }
$$ 


\section{B. Energy Model for one and p parallel processor(s):}

When executing a parallel application, total energy consumption can be divided into four parts: computation energy, $E_{c}$ main memory access energy, $E_{\text {mem }}, \mathrm{I} / \mathrm{O}$ access energy, $E_{I O}$, and other system components energy, $E_{\text {other }}$, such as motherboard, system and CPU fans, power supply, etc. Thus, we have total energy $E[20]$ :

$$
E=E_{c}+E_{\text {mem }}+E_{I O}+E_{\text {other }}
$$

The first three parts of this equation can be further separated into two energy states: running state and idle state. For example, $E_{c}$ can be divided into $E_{c-o n}$ and $E_{c-i d l e}$. Thus, we can deduce total energy $E$ as $[18,20]$ :

$$
\begin{aligned}
& E=E_{c-o n}+E_{c-i d l e}+E_{\text {mem-on }}+E_{\text {mem-idle }}+ \\
& E_{I O-\text { on }}+E_{I O-\text { idle }}+E_{\text {other }}
\end{aligned}
$$

From(6) and (8),

$$
E=\alpha T P_{\text {total-idle }}+W_{c} t_{c} \Delta P_{c}+W_{m} t_{m} \Delta P_{m}+T_{I O} \Delta P_{I O}(9)
$$

where $W_{c} t_{c}$ is the total computation time; $W_{m} t_{m}$ is the total memory access time and $T_{I O}$ is the total $\mathrm{I} / \mathrm{O}$ access time.

$$
\begin{gathered}
\Delta P_{c}=P_{c-o n}-P_{c-i d l e} \\
\Delta P_{m}=P_{m-o n}-P_{m-i d l e} \\
\Delta P_{I O}=P_{I O-o n}-P_{I O-i d l e}
\end{gathered}
$$

Equation (9) seems quite cumbersome; however, it is intuitive: $\alpha T P_{\text {total-idle }}$ is the total energy consumption of an idle-state system during an application's execution time. $W_{c} t_{c} \Delta P_{c}$ is the additional energy used while an application is performing computation. Similarly, $W_{m} t_{m} \Delta P_{m}$ and $T_{I O} \Delta P_{I O}$ are the additional energy consumption for conducting main memory and $\mathrm{I} / \mathrm{O}$ accesses.

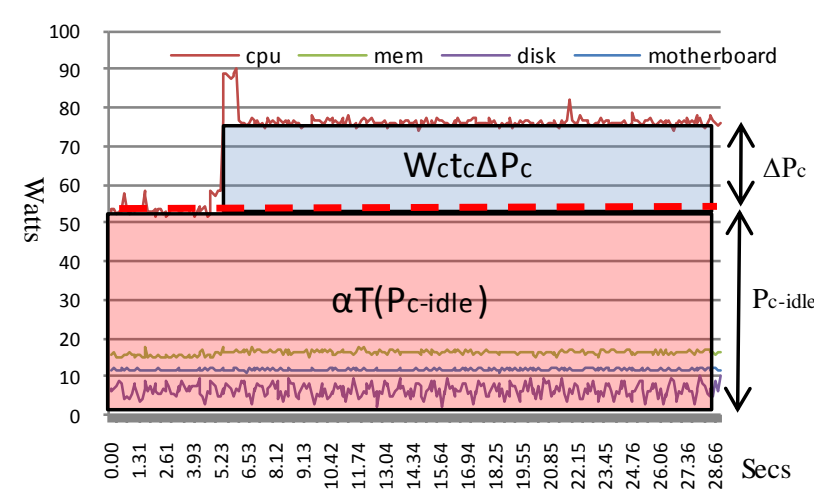

Figure 10. Power Profiling of MPI_FFT program in HPCC Benchmark

Figure 10 provides additional insight to Equation (9). It shows the power profiling of the MPI_FFT program in the HPC Challenge Benchmark [19] measured by the
PowerPack framework. The power fluctuates for each component over the idle-state power line (dashed line) during the execution time. For the CPU, the red shaded (lower) portion in Figure 10 represents total CPU energy consumption in idle-state, and the blue (upper) portion represents the additional energy while doing computation.

In reality, $\mathrm{I} / \mathrm{O}$ access time includes the network and all kinds of local storage devices accesses. If an application is disk I/O-intensive, it should introduce $T_{\text {disk }}$ to the performance and the energy model. For simplicity, we assume a simple, flat model for $\mathrm{I} / \mathrm{O}$ accesses though the benchmarks we measured did not exercise $\mathrm{I} / \mathrm{O}$ making this component effectively zero. Users can always replace $T_{I O} \Delta P_{I O}$ with any combinations of specific $\mathrm{I} / \mathrm{O}$ components according to their parallel applications. Demonstrating the accuracy of the model for all types of $\mathrm{I} / \mathrm{O}$ is beyond the scope of this paper and the subject of future work.

The equations follow similar to Equation (6):

$T^{\prime}=\alpha T=\alpha\left(W_{c} t_{c}+W_{m} t_{m}+T_{n e t}\right)$

With energy model:

$$
\begin{aligned}
& E=\alpha T P_{\text {total-idle }}+W_{c} t_{c} \Delta P_{c}+W_{m} t_{m} \Delta P_{m}+ \\
& T_{\text {net }}\left(P_{\text {net-on }}-P_{\text {net-idle }}\right)
\end{aligned}
$$

In our experiments (on both the Dori system with Ethernet and SystemG with InfiniBand), the difference between $P_{\text {net-on }}$ and $P_{\text {net-idle }}$ is not significant so we simply ignore the effect $T_{\text {net }}\left(P_{\text {net-on }}-P_{\text {net-idle }}\right)$ in (11):

$$
E=\alpha T\left(P_{\text {total-idle }}\right)+W_{c} t_{c} \Delta P_{c}+W_{m} t_{m} \Delta P_{m}
$$

\section{Energy Model for A Single Processor}

Equations (10) and (12) are the kernel components of the performance model and iso-energy-efficiency model in this paper. Let us apply these to $E_{1}$ which we discussed in Section 3. When an application executes on a single processor, there are no messages exchanged. This means no $T_{\text {net }}$ in (10). Thus, $E_{1}$ becomes:

$$
\begin{aligned}
& E_{1}=\alpha T_{1}\left(P_{\text {total-idle }}\right)+W_{c} t_{c} \Delta P_{c}+W_{m} t_{m} \Delta P_{m} \\
& \text { where } T_{1}=\left(W_{c} t_{c}+W_{m} t_{m}\right)
\end{aligned}
$$

\section{Energy Model for p Parallel Processors}

Similarly, to get $E_{p}$, we define the energy model in $i$ th $(1 \leq i \leq p)$ processor among $p$ parallel processors:

$$
\begin{aligned}
& E_{p_{\_} i}=\alpha T_{p_{-} i}\left(P_{\text {total-idle }}\right)+\left(w_{c_{-} i}+w_{c o_{-} i}\right) t_{c} \Delta P_{c}+ \\
& \left(w_{m_{-} i}+w_{m_{-} i}\right) t_{m} \Delta P_{m} \\
& \text { where } T_{p_{-} i}=\left[\left(w_{c_{-} i}+w_{c o_{-} i}\right) t_{c}+\left(w_{m_{-} i}+w_{m o_{-} i}\right) t_{m}+T_{\text {net } \_}\right]
\end{aligned}
$$

In (14), $w_{c o_{-} i}$ and $w_{m o_{-} i}$ are computation and memory access overheads for the $i$ th of $p$ processors in terms of 
parallelism. Thus, we have $E_{p}$ representing total energy consumption for all processors:

$$
\begin{gathered}
E_{p}=\sum_{i=1}^{p} E_{p_{-} i}=\alpha T_{p}\left(P_{\text {total-idle }}\right)+\left(W_{c}+\right. \\
\left.W_{c o}\right) t_{c} \Delta P_{c}+\left(W_{m}+W_{m o}\right) t_{m} \Delta P_{m}
\end{gathered}
$$

where, $T_{p}=\sum_{i=1}^{p} T_{p_{i} i}=\left[\left(W_{c}+W_{c o}\right) t_{c}+\left(W_{m}+W_{m o}\right) t_{m}+\right.$ $\left.\sum_{i=1}^{p} T_{\text {net_i}}\right]$ and capital "W" represents summation of all workload in all processors.

From (1) we can calcu late the energy overhead $E_{o}$ :

$$
\begin{aligned}
& \quad E_{o}=E_{p}-E_{1}=\alpha T_{o}\left(P_{\text {total-idle }}\right)+W_{c o} t_{c} \Delta P_{c}+ \\
& W_{m o} t_{m} \Delta P_{m}
\end{aligned}
$$

In (15) and (16), $W_{c o}$ is the total parallel computation overhead $\left(W_{c o}=\sum_{i=1}^{p} w_{c o \_} i\right)$ and $W_{m o}$ represents the total parallel memory access overhead $\left(W_{m o}=\sum_{i=1}^{p} w_{m o \_}\right)$. $\sum_{i=1}^{p} T_{n e t_{-} i}$ stands for accumulated networking time. $\sum_{i=1}^{p} T_{n e t_{-} i}$ can be further divided into two parts: message start up time and data transmitting time [32]. Communication overhead modeling varies depending on application and network infrastructure. Equation (17) is a general approach and specific parameterization for network modeling is applied for each application (see Section 5).

$$
\sum_{i=1}^{p} T_{n e t_{-} i}=M t_{m s g}+B t_{\text {Byte }}
$$

So that $E_{o}$ can be expressed as:

$E_{o}=\alpha T_{o} P_{\text {total-idle }}+W_{c o} t_{c} \Delta P_{c}+W_{m o} t_{m} \Delta P_{m}$ where $T_{o}=\left(W_{c o} t_{c}+W_{m o} t_{m}+M t_{m s g}+B t_{\text {Byte }}\right)$

\section{E. Energy Efficiency Factor (EEF)}

Using the Equations (13) and (18), we can formulate the Energy Efficiency Factor (EEF) more accurately,

$$
\begin{aligned}
E E F=\frac{E_{o}}{E_{1}} & =\frac{\alpha T_{o}\left(P_{\text {total-idle }}\right)+W_{c o} t_{c} \Delta P_{c}+W_{m o} t_{m} \Delta P_{m}}{\alpha T_{1}\left(P_{\text {total-idle }}\right)+W_{c} t_{c} \Delta P_{c}+W_{m} t_{m} \Delta P_{m}} \\
\text { Where } T_{o} & =\left(W_{c o} t_{c}+W_{m o} t_{m}+M t_{m s g}+B t_{B y t e}\right) \\
T_{1} & =\left(W_{c} t_{c}+W_{m} t_{m}\right)
\end{aligned}
$$

Equation (19) contains two categories of parameters which directly impact performance and energy consumption: 1) machine dependent variables $t_{c}, t_{m}, t_{m s g}, t_{\text {Byte }}, P_{\text {total-idle }}, \Delta P_{c}, \Delta P_{m}$. and 2) application dependent variables: $\alpha, W_{c}, W_{m}, W_{c o}, W_{m o}, M$, and $B$. For the application dependent vector, $V_{a p p}$, the processor number $p$ and problem size $n$ are two main factors affecting these parameters. They can be represented as $V_{a p p}(p, n)=$ $\left(\alpha, W_{c}, W_{m}, W_{c o}, W_{m o}, M, B\right)$.
The values of $W_{c}, W_{m}, W_{c o}, W_{m o}$ can be obtained by the combination of analyzing an application's algorithm and directly measuring the specific performance counters to estimate the on-off chip workload. Also, $M$ and $B$ can be acquired by using PMPI in MPICH2 [30] or TAU[38]. The overlap factor $\alpha$ can be calculated using:

$$
\alpha=\frac{\text { actual execution time }}{W_{c} t_{c}+W_{m} t_{m}+T_{\text {net }}}
$$

The mach ine dependent vector can be represented as:

$$
\begin{aligned}
& V_{\text {machine }}\left(f, N_{\text {bandwidth }}\right) \\
& =\left(t_{c}, t_{m}, t_{m s g}, t_{\text {Byte }}, P_{\text {total-idle }}, \Delta P_{c}, \Delta P_{m}\right)
\end{aligned}
$$

For machine dependent variables, machine frequency, $f$ and the network bandwidth, $N_{\text {bandwidth }}$, are the main factors affecting these parameters. For the time parameters, $t_{c}$ is $\frac{C P I_{o n}}{f}, t_{m}, t_{m s g}$ can be also described as functions of $f$. Only $t_{\text {Byte }}$ is related with the network bandwidth. From Kim et al $[6,34]$ :

$$
\begin{aligned}
& f_{\text {max }} \propto \frac{\left(V_{d d}-V_{t h}\right)^{\beta}}{V_{d d}} \\
& P=P_{d y n}+P_{\text {leak }}=A C V_{d d}^{2} f+I_{\text {leak }} V_{d d}
\end{aligned}
$$

We can assume $P_{\text {total }-i d l e}, \Delta P_{c}, \Delta P_{m}$, are also functions of $f$. Here we assume power is proportional to $f^{\gamma}(\gamma \geq 1)$. We use the correlation between power and frequency in our energy model to predict total energy consumption and energy efficiency of large scale parallel system.

From Equations (2) and (19), the iso-energy-efficiency model for parallel applications can be defined as:

$$
\begin{gathered}
E E=\frac{1}{1+E E F}=\frac{1}{1+\frac{\alpha T_{o}\left(P_{\text {total-idle }}\right)+W_{c o} t_{c} \Delta P_{c}+W_{m o} t_{m} \Delta P_{m}}{\alpha T_{1}\left(P_{\text {total-idle }}\right)+W_{c} c_{c} \Delta P_{c}+W_{m} t_{m} \Delta P_{m}}} \\
\text { where } \mathrm{T}_{\mathrm{o}}=\left(\mathrm{W}_{\mathrm{co}} \mathrm{t}_{\mathrm{c}}+\mathrm{W}_{\mathrm{mo}} \mathrm{t}_{\mathrm{m}}+\mathrm{Mt}_{\mathrm{msg}}+\mathrm{Bt}_{\mathrm{Byte}}\right) \\
\mathrm{T}_{1}=\left(\mathrm{W}_{\mathrm{c}} \mathrm{t}_{\mathrm{c}}+\mathrm{W}_{\mathrm{m}} \mathrm{t}_{\mathrm{m}}\right)
\end{gathered}
$$

In equation (21), EEF is a combination of machine and application dependent parameters. To maximize the system energy efficiency, we need to keep EEF as small as possible by scaling characteristics such as degree of parallelis $\mathrm{m}$, workload, proces sor frequencies and network bandwidth.

\section{F. Computational Overlap}

Accurately capturing performance characteristics is critical to a model of iso-energy-efficiency. Early on in our attempt to create an iso-energy-efficiency model we realized computational overlap, or the ability to conduct computations while waiting on memory or communication delays, could not be ignored since they can reduce execution time dramatically [37]. The amount of overlap varies with an application, the underlying machine architecture, and compiler settings. For example, an application code may have computation time $O\left(n^{2}\right)$, memory access time $O(n)$ and network transmitting time $O(n)$. Without 
optimization, the total execution time is $O\left(n^{2}+n+n\right)$; however, the actual time is smaller.

Thus we propose a comprehensive optimization parameter, $\alpha$, to capture computational overlap. $\alpha$ Theoretical execution time consists of computation time, me mory access time, and remote data access time (or network transmission time). Thus we have:

Theoretical Execution Time $=$ Computation Time + Memory Access Time + Network Transmission Time

And we can define $\alpha$ :

$$
\alpha=(\text { Actual Execution Time) / (Theoretical Execution Time) }
$$

For parallel applications, we found empirically for the applications studied that an application using the same compiler settings has the same $\alpha$ value under different levels of parallelis m. However, different applications could have different $\alpha$ due to different execution patterns. In addition, same applications running on different machines also have different $\alpha$ values because of diverse underlying architectures.

\section{CONCLUSIONS}

In this paper, we present a system level energy efficiency model for various parallel applications and large scale parallel system architectures. We extend the concept of performance isoefficiency to iso-energy-efficiency and show how to build an accurate system level energy efficiency model step by step. Then we apply our analytical model to real scientific applications from NAS Parallel Benchmark suites and illustrate how to derive essential model parameters to predict total system energy consumption and efficiency for large scaling parallel systems. After a thorough and detailed investigation of machine and application dependent parameters which have nontrivial impact on system energy efficiency, we apply the model to three scientific benchmarks representing different execution patterns to study what the influential factors are for system energy efficiency and how to scale them to maintain efficiency. The results conducted on two power-aware clusters show that our model can predict total system energy cons umption within average $5 \%$ prediction error rate for parallel applications with various execution and communication patterns. And also, in the case study experiments, the results clearly show what the most influential factors are and how these factors can be tuned to maintain energy efficiency. Though this model can precisely predict energy in various combinations of applications and hardware architecture, we still have a bit more parameters compared with the micro-architecture approach. In the future, we plan to integrate PowerPack with other system measurement tools and together make it more compatible and easier for all users to model. Also, we want to extend the current model to heterogeneous systems.

\section{REFERENCES}

[1] (2010). US department of energy annual report. Available: http://www.eia.doe.gov/

[2] V. W. Freeh, F. Pan, N. Kappiah, D. K. Lowenthal, and R. Springer, "Exploring the Energy-Time Tradeoff in MPI Programs on a Power-Scalable Cluster," presented at the Proceedings of the 19th IEEE International Parallel and Distributed Processing Symposium (IPDPS'05) Papers - Volume 01, 2005.

[3] R. Ge, X. Feng, and K. W. Cameron, "Improvement of Power-Performance Efficiency for High-End Computing," presented at the Proceedings of the 19th IEEE International Parallel and Distributed Processing Symposium (IPDPS'05) - Workshop 11 - Volume 12, 2005.

[4] R. Ge, X. Feng, and K. W. Cameron, "Performanceconstrained Distributed DVS Scheduling for Scientific Applications on Power-aware Clusters," presented at the Proceedings of the $2005 \mathrm{ACM} / \mathrm{IEEE}$ conference on Supercomputing, 2005.

[5] V. W. Freeh and D. K. Lowenthal, "Using multiple energy gears in MPI programs on a power-scalable cluster," presented at the Proceedings of the tenth ACM SIGPLAN symposium on Principles and practice of parallel programming, Chicago, IL, USA, 2005.

[6] J. Li and J. F. Martinez, "Dynamic power-performance adaptation of parallel computation on chip multiprocessors," in The Twelfth International Symposium on High-Performance Computer Architecture, 2006, pp. 77-87.

[7] A. Y. Grama, A. Gupta, and V. Kumar, "Isoefficiency: measuring the scalability of parallel algorithms and architectures," in Multiprocessor performance measurement and evaluation, ed: IEEE Computer Society Press, 1995, pp. 103-112.

[8] (2010). The NAS Parallel Benchmarks. Available: http://www.nas.nasa.gov/Resources/Software

[9] G.M. Amdahl, "Validity of the Single Processor Approach to Achieving Large-Scale Computing Capabilities," AFIPS Spring Joint Computer Conference, Reston, VA, 1967.

[10] A. Y. Grama, A. Gupta, V. Kumar, and G. Karypis, Introduction to Parallel Computing, 2 ed.: The AddisionWesley Longman Publishing, 2003.

[11] J. L. Gustafson, "Reevaluating Amdahl's law," Commun. ACM, vol. 31, pp. 532-533, 1988.

[12] X.-H. Sun and L. M. Ni, "Scalable problems and memory-bounded speedup," J. Parallel Distrib. Comput., vol. 19, pp. 27-37, 1993.

[13] M. D. Hill and M. R. Marty, "Amdahl's law in the multicore era," IEEE Computer, 2008.

[14] J. M. Paul and B. H. Meyer, "Amdahl's law revisited for single chip sy stems," Int. J. Parallel Program., vol. 35, pp. 101-123, 2007.

[15] (2010). "TOP500 Supercomputing Sites". Available: http://www.top500.org

[16] W. Feng and K.Cameron, "The Green500 List: Encouraging Sustainable Supercomputing" Computer, vol. 40, p. 50_55, 2007.

[17] (2008). The SPEC Power benchmark. Available: http://www.spec.org/power_ssj2008/ 
[18] S. Song, R. Ge, X. Feng, and K. W. Cameron, "Energy Profiling and Analysis of the HPC Challenge Benchmarks," International Journal of High Performance Computing Applications, vol. 23, pp. 265-276, 2009.

[19] P. R. Luszczek, D. H. Bailey, J. J. Dongarra, J. Kepner, R. F. Lucas, R. Rabenseifner, et al., "The HPC Challen ge (HPCC) benchmark suite," presented at the Proceedings of the 2006 ACM/IEEE conference on Supercomputing, Tampa, Florida, 2006.

[20] R. Ge, X. Feng, S. Song, and K. W. Cameron, "PowerPack: Energy Profiling and Analysis of HighPerformance Systems and Applications," IEEE Transactions on Parallel and Distributed Sy stems, vol. 99, pp. 658-671, 2009.

[21] K. W. Cameron, R. Ge, and X. Feng, "High-Performance, Power-Aware Distributed Computing for Scientific Applications," Computer, vol. 38, pp. 40-47, 2005.

[22] X. Feng, R. Ge, and K. W. Cameron, "Power and Energy Profiling of Scientific Applications on Distributed Systems," presented at the Proceedings of the 19th IEEE International Parallel and Distributed Processing Sy mposium (IPDPS'05) - Papers - Volume 01, 2005.

[23] R. Springer, D. K. Lowenthal, B. Rountree, and V. W. Freeh, "Minimizing execution time in MPI programs on an energy-constrained, power-scalable cluster," presented at the Proceedings of the eleventh ACM SIGPLAN symposium on Principles and practice of parallel programming, New York, New York, USA, 2006.

[24] V. W. Freeh and D. K. Lowenthal, "Using multiple energy gears in MPI programs on a power-scalable cluster," presented at the Proceedings of the tenth ACM SIGPLAN symposium on Principles and practice of parallel programming, Chicago, IL, USA, 2005.

[25] R. Ge and K. W. Cameron, "Power-Aware Speedup," in proceedings of the 21st IEEE International Parallel and Distributed Processing Sy mposium, 2007, pp. 56-56.

[26] N. Jiang, J. Pisharath, and A. Choudhary, "Characterizing and improving energy-delay tradeoffs in heterogeneous communication systems," Signals, Circuits and Systems, 2003. SCS 2003. International Sy mposium vol. 2, pp. 409-412, 2003.

[27] Y. Ding, K. Malkowski, P. Raghavan, and M. Kandemir, "Towards Energy Efficient Scaling of Scientific Codes," in IEEE International Symposium on Parallel and Distributed Processing, 2008, pp. 1 - 8

[28] D. A. Patterson and J.L.Hennessy, Computer Architecture: A quantitative approach, 3rd ed. San Francisco, CA: Morgan Kaufmann Publishers, 2003.

[29] (2010). LMbench - Tools for Performance Analysis. Available: http://www.bitmover.com/lmbench/

[30] MPICH2: high-performance and widely portable implementation of the Message Passing Interface (MPI) standard.

Available: http://phase.hpcc.jp/mirrors/mpi/mpich2/

[31] InfiniBand Trade Association. Available: http://www.infinibandta.org/

[32] J. Pjeivac-Grbovi, T. Angskun, G. Bosilca, G. E. Fagg, E. Gabriel, and J. J. Dongarra, "Performance analysis of MPI collective operations," Cluster Computing, vol. 10, pp. 127-143, 2007.
[33] R. Thakur, "Improving the performance of collective operations in MPICH," in Recent Advances in Parallel Virtual Machine and Message Passing Interface. Number 2840 in LNCS, ed: Springer Verlag, 2003, pp. 257-267.

[34] N. S. Kim, T. Austin, D. Blaauw, T. Mudge, Kriszti, n. Flautner, et al., "Leakage Current: Moore's Law Meets Static Power," Computer, vol. 36, pp. 68-75, 2003.

[35] K. Choi, R. Soma, and M. Pedram, "Dynamic voltage and frequency scaling based on workload decomposition," presented at the Proceedings of the 2004 international symposium on Low power electronics and design, Newport Beach, California, USA, 2004.

[36] Q. Wu, M. Martonosi, D. W. Clark, V. J. Reddi, D. Connors, Y. Wu, et al., "A Dynamic Compilation Framework for Controlling Microprocessor Energy and Performance," presented at the Proceedings of the 38th annual IEEE/ACM International Symposium on Microarchitecture, Barcelona, Spain, 2005.

[37] K. C. Louden, Compiler Construction: Principles and Practice, 1st edition, 997.

[38] (2010). TAU: Tuning and Analysis Utilities. Available: http://www.cs.uoregon.edu/research/tau/home.php 